\title{
Maintenance Optimization of a 2-Component Swappable Series System Using the Delay-Time Concept
}

\author{
Liying wang $\mathbb{D}$ and Wenhua Zhang $\mathbb{D}$ \\ Department of Mathematics \& Physics, Shijiazhuang Tiedao Institute, Shijiazhuang, China \\ Correspondence should be addressed to Liying wang; wly_sjz@sohu.com
}

Received 20 April 2021; Revised 2 June 2021; Accepted 15 July 2021; Published 26 July 2021

Academic Editor: Alessandro Rasulo

Copyright (c) 2021 Liying wang and Wenhua Zhang. This is an open access article distributed under the Creative Commons Attribution License, which permits unrestricted use, distribution, and reproduction in any medium, provided the original work is properly cited.

In the 2-component swappable series system, the two components undertake tasks with different loads and degrade at different speeds. To prolong the lifetime of the series system, these two components are swapped in the operating process of the system in practice. This is common in the maintenance of duplexing steelmaking systems, tires of vehicles, and steel rails in curves. The failure process of each component in the system is modeled based on a two-stage delay-time concept and divided into two stages: normal and defective. Inspections are carried out periodically on the system. Two components may be swapped once at an inspection time that the two components are both in the normal stage. Due to the increase or decrease of loads, normal and defective time distributions after the swap are assumed to be different from those prior to the swap. The system is subjected to failure, inspection, and age-based renewals. The number of inspections over the maximum usage time of the system and the swap time are optimized jointly by minimizing the expected cost per unit time in a long run. A numerical example is presented to demonstrate the model.

\section{Introduction}

The motivation of the paper comes from the operating process of the duplexing steelmaking system and maintenance of vehicles and steel rails in curves. The duplexing steelmaking system usually consists of two converters. One converter is for dephosphorization, and the other is for decarburization. Since each converter can fail mainly due to one failure mode, we treat them as components. Due to the heavier load, the decarburization converter degrades more quickly than the dephosphorization one. To prolong the system's lifetime, the decarburization converter will be used for dephosphorization at a later stage of the furnace life; that is, the two converters are swapped. The swap time is usually determined by engineers according to their experiences. The swap can also be found in the process of vehicle maintenance. For a front drive vehicle, the tire wear of front and rear wheels is different. To keep the safety, the handling, and the stability, they are swapped periodically according to the handbook of vehicle maintenance. The swap number and times are also mapped out by original equipment manufacturers. In a curve, the outside steel rails degrade more quickly than the inside ones; they are also swapped periodically in the maintenance of railway lines. Some literatures studied maintenance policies with the swap. Taking advantage of the different degradation rates of batteries, Almuhtady et al. [1] introduced a policy incorporating implementation of swapping and substitution actions. Fu et al. [2] studied a new maintenance policy, which performs periodic preventive system replacements and periodic preventive component reallocations between system replacements as well as minimal repairs for emergency failures. Sun et al. [3] quantified the benefit of incorporating the reallocation into the condition-based maintenance.

The delay-time concept was proposed by Christer [4]. The failure process in the delay-time concept is divided into two/three stages, named normal and defective/normal, minor defective and major defective (Christer and Waller [5]; Wang [6]; Wang [7]; Wang et al. [8]; and Yang [9]). The 
defective stages are not self-announcing and they can be identified by inspections. When the component is detected to be in a defect state at the detection time, the component will be repaired. Delay-time-based maintenance models can capture the relationship between the number of failures and inspection intervals, so they are widely used in describing the degradation of plants and optimizing of inspection intervals (Baker and Wang [10]; Baker and Christer [11]; Jia and Christer [12]; Wang [13]; Aven and Castro [14]; Jones et al. [15]; and Scarf et al. [16]). Single-component and multicomponent models have been proposed in the literature (Wang et al. [17]; Lu et al. [18]; and Liu et al. [19]). An adjustable inspection and replacement strategy for systems subject to 0-1 logic failure and two-stage failure process was proposed by Ma et al. [20]. Peng et al. [21] constructed a single-unit system subject to two types of failures: a traditional catastrophic failure and a two-stage delayed failure. Maintenance strategy considering imperfect inspections is discussed in their paper. Yang et al. [22] considered the optimization of a preventive maintenance policy based on dependent two-stage deterioration and external shocks. For systems experiencing internal failures and external shocks, the effects of external shocks were discussed as two stages of random virtual age increment, and a mission abort strategy was proposed (Qiu and Cui [23]). Zhao et al. [24] investigated the joint optimization of inspection and condition-based mission abort policies for systems subject to continuous degradation. A new model whose degradation was described by dependent two-stage failure process with competing failures was built by Qiu and Cui [25]. Heydari [26] gave a periodic inspection policy for products with extended warranty period-based two-stage failure process. Based on three-stage failure process, Wang et al. [27] proposed a condition-based maintenance policy for products sold with renewal warranty contract.

Due to the advantage mentioned above, we built the 2component swappable series system using the delay-time concept in this paper. The new contributions of this paper are as follows. By using the concept of delay time, the failure process of each component is divided into normal and defect stages, which can be detected by inspections. The two components can be swapped once at a detection time. Inspection renewals are adopted at detection times depending on the states of the two components. So, inspection and swapping are incorporated into maintenance in this paper. By jointly optimizing the number of inspections and swap time, long-term expected cost per unit time is minimized. It is worth noting that the cost of swapping components is usually much lower than the cost of replacing them, so when the workload of each component is unbalanced, swap with replacement can further reduce the cost of running the system.

The remaining part of the paper is organized as follows. Section 2 provides the modeling assumptions and notations. Section 3 establishes the probabilities of failure renewal, inspection renewal, and the overhaul. Section 4 models the long run expected cost per unit time. Section 5 presents a numerical example. Section 6 concludes the paper and suggests some future works.

\section{Modeling Assumptions and Notations}

We consider a series system with two components, namely, components 1 and 2 .

(1) The two components undertake the same tasks but with different loads. So, they degrade at different speeds, and their lifetimes are statistically different. The failure process of each component is divided into two stages, normal and defective stages. The distributions in different stages and for different components are statistically independent.

(2) The maximum usage time of the system is $T_{\text {age }}$. The system is subjected to an overhaul (replace both components) when it reaches $T_{\text {age }}$; no failure occurs and no defect is found before $T_{\text {age }}$. Once a component fails, both components are repaired or replaced, which can be regarded as the failure renewal of the system. Note that both components are replaced at $T_{\text {age }}$. While on a failure renewal, a component fails and the other component may be in normal or defective states; it can be restored at a lower cost. Furthermore, the usage times of the two components on a failure renewal are shorter than those at $T_{\text {age; }}$; they can also be restored at a lower cost. So, we assume that the cost of an overhaul is greater than that of a failure renewal.

(3) The system is subjected to equispaced inspections in $\left(0, T_{\text {age }}\right) . M$ is the number of inspections in $\left(0, T_{\text {age }}\right)$, and it is a decision variable. Let $T=T_{\text {age }} /(M+1)$. Then, $T$ is the inspection interval and both components of the system are inspected at times $k T(k=1,2, \ldots, M)$. Inspections are perfect. The downtime due to inspections is negligible compared with the inspection intervals or inspections can be performed online, so there is no downtime. However, the costs of inspections are considered.

(4) The two components are swapped once at time $\tau=m^{\prime} T\left(m^{\prime} \in\{1,2, \ldots, M\}\right)$ provided the system has not failed before $\tau$ and the no defect is found before $\tau$. Due to the variety of the load, the hazard function of normal time distributions and the delay time distribution will be different from those prior to the swap. Assume that extra cost is incurred for the swap.

(5) If one or two components are found to be in the defective state by an inspection, the two components are repaired or replaced, which can be regarded as the inspection renewal of the system.

Assumptions (1) and (3) can be justified in previous delay time modeling applications (Wang [6]; Wang et al. [8]). According to assumption (2), $T_{\text {age }}$ in this paper is the maximum usage time of the system. It is fixed, instead of a decision variable. The simultaneous optimization of $(M, T, \tau)$ in the case that $T_{\text {age }}$ is assumed to be the threshold of age-based renewal will be conducted in the future.

To reduce the cost of system startup and shutdown, we assume that the swap is carried out at inspection time in 
assumption (4). This is a common case in practice. Furthermore, due to the swap, the component that takes lighter loads prior to the swap will take heavier ones after the swap, so it will degrade more quickly than itself in the case that swap is not carried out. On the contrary, the component will degrade more slowly than another component in the case that swap is not carried out. So, the assumptions about hazard functions in assumption (4) are reasonable. As stated in assumption (4), the two components are swapped only once in this paper; more than one swap may happen in practice. This will be left to a separate paper.

The explanations of assumption (5) are as follows. If one component is found to be in the defective state or fails, there could be an opportunity to check another component and conduct repair or replacement. This assumption can be relaxed with more mathematics involved, but now we keep in for simplicity.

We will use the following notations in subsequent modeling:

$X_{i}$ is the random time of the normal stage of component $i(i=1,2)$

$\lambda_{i}(t)$ is the hazard function of component $i(i=1,2)$ in the normal stage prior to the swap

$\tilde{\lambda}_{i}(t)$ is the hazard function of component $i(i=1,2)$ in the normal stage after the swap

$F_{i}(t)$ is the cumulative distribution function (cdf) of $X_{i}(i=1,2)$

$f_{i}(t)$ is the probability density function (pdf) of $X_{i}(i=1,2)$

$Y_{i}$ is the delay time of component $i$ prior to the swap

$g_{i}(t)$ is the pdf of $Y_{i}(i=1,2)$

$G_{i}(t)$ is the cdf of $Y_{i}(i=1,2)$

$\tilde{Y}_{i}$ is the delay time of component $i(i=1,2)$ after the swap

$\tilde{g}_{i}(t)$ is the pdf of $\tilde{Y}_{i}(i=1,2)$.

$\widetilde{G}_{i}(t)$ is the cdf of $\widetilde{Y}_{i}(i=1,2)$

$T$ is the interval of inspections

$M$ is the number of inspections

$T_{\text {age }}$ is the maximum usage time of the system

$\tau$ is the time of the swap

$T_{f}$ is the random time that the system fails in the case that the two components are not swapped

$T_{1 d}$ is the random time that the system is renewed due to the defect of component 1 or component 2 in the case that the two components are not swapped

$T_{2 d}$ is the random time that the system is renewed due to the defects of components 1 and 2 in the case that the two components are not swapped

$T_{s f}$ is the random time that the system fails in the case that the two components are swapped

$T_{s 1 d}$ is the random time that the system is renewed due to the defect of component 1 or 2 in the case that the two components are swapped
$T_{s 2 d}$ is the random time that the system is renewed due to the defect of component 1 and 2 in the case that the two components are swapped

$Z$ is the failure renewal time

$f_{m}(z)$ is the probability density function of the time that $Z$ exceeds the last inspection in the case that $T_{f} \in((m-1) T, m T)$ (before the swap, $\left.z \in(0, T)\right)$

$\widetilde{f}_{m}(z)$ is the probability density function of the time that $Z$ exceeds the last inspection in the case that $T_{f} \in((m-1) T, m T)$ (after the swap, $z \in(0, T)$ )

$C_{i}$ is the average cost per inspection

$C_{f}$ is the average cost per failure renewal

$C_{1 d}$ is the average cost per inspection renewal in the case that one component of the system is identified to be in defective state

$C_{2 d}$ is the average cost per inspection renewal in the case that two components of the system are identified to be in defective state

$C_{s}$ is the average cost per swap

$C_{a}$ is the average cost of the overhaul

According to the assumptions and notations above, the survival function of component $i(i=1,2)$ in the normal stage:

$$
1-F_{i}(t)= \begin{cases}\exp \left\{-\int_{0}^{t} \lambda_{i}(u) \mathrm{d} u\right\}, & t \leq \tau, \\ \exp \left\{-\int_{0}^{\tau} \lambda_{i}(u) \mathrm{d} u-\int_{\tau}^{t} \widetilde{\lambda}_{i}(u) \mathrm{d} u\right\}, & t>\tau .\end{cases}
$$

So, the cdf of $X_{i}(i=1,2), F_{i}(t)$, can be determined by $\lambda_{i}(t)(i=1,2)$ and $\bar{\lambda}_{i}(t)$. From equation (1), the pdf of $X_{i}(i=1,2)$

$$
f_{i}(t)= \begin{cases}\lambda_{i}(t) \exp \left\{-\int_{0}^{t} \lambda_{i}(u) \mathrm{d} u\right\}, & t \leq \tau, \\ \tilde{\lambda}_{i}(t) \exp \left\{-\int_{0}^{\tau} \lambda_{i}(u) \mathrm{d} u-\int_{\tau}^{t} \tilde{\lambda}_{i}(u) \mathrm{d} u\right\}, & t>\tau .\end{cases}
$$

The decision variables in this paper are the number of inspections $M$ and the swap time $\tau$. The objective is to minimize the long run expected cost per unit time.

\section{Probabilistic Models for Failure, Defect Stage Identifications}

We use the long run expected cost per unit time as a measure to optimize the number of inspections $M$ and the swap time $\tau$. In order to derive the long run expected cost per unit time, the renewal probabilities prior to and after the swap need to be formulated. There are four mutually exclusive scenarios at the end of a renewal cycle, that is, a failure renewal, an overhaul, inspection renewals due to one or two components' defects. 


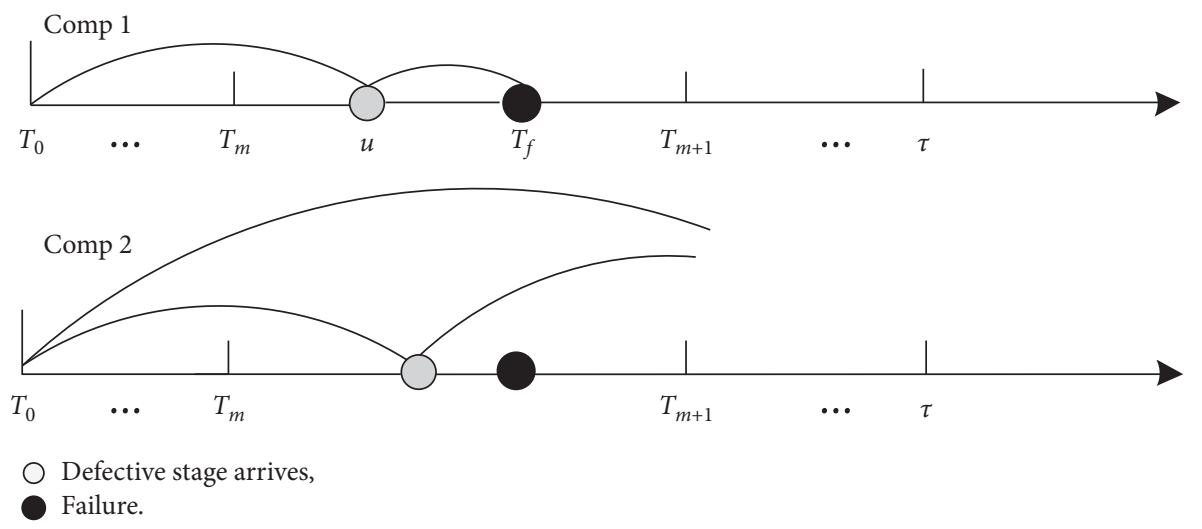

FIGURE 1: The system fails before any defective stage of the two components is identified by the inspection. ○ denotes defective stage arrives; - denotes failure.
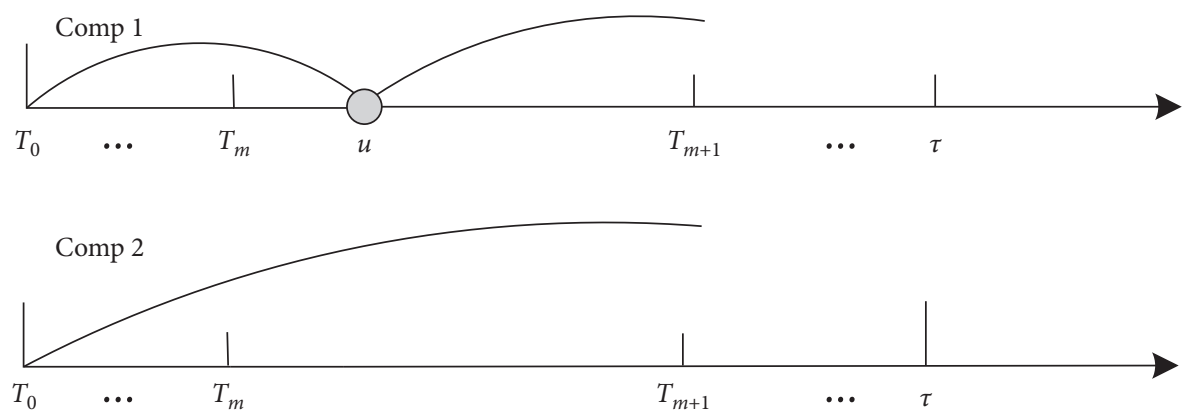

(a)
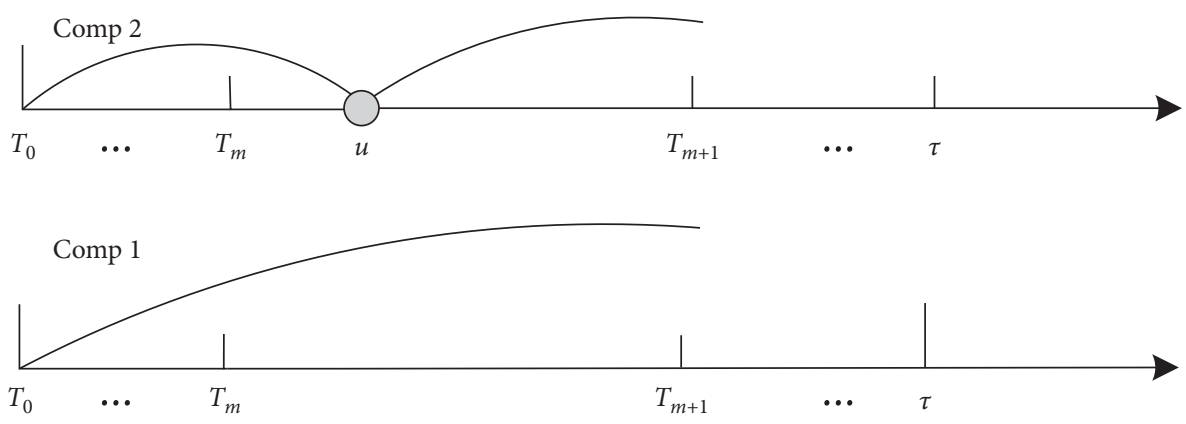

(b)

Figure 2: Illustration of a renewal due to the defect of one component. (a) Component 1 is defective; component 2 is normal. (b) Component 2 is defective; component 1 is normal.
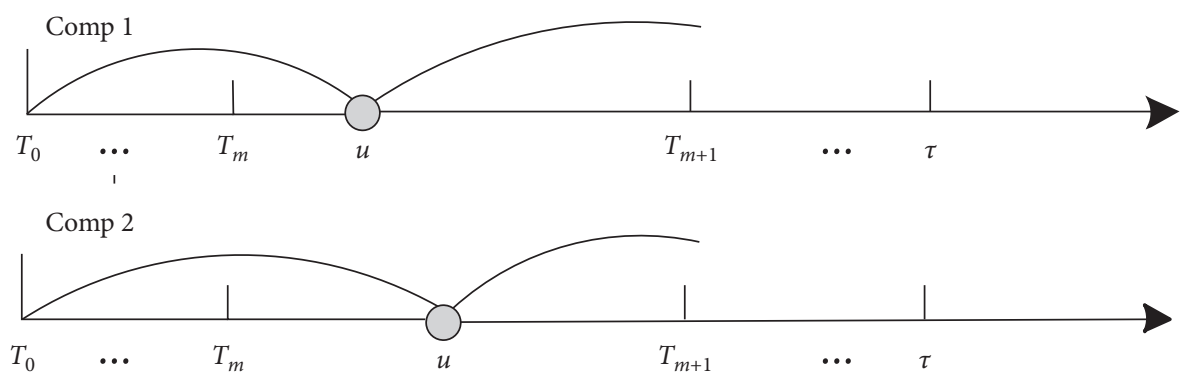

FIGURE 3: Illustration of a renewal due to defects of two components. 


\subsection{Probability of a Renewal Prior to the Swap}

(1) The system fails at $T_{f} \quad\left(T_{f} \in(m T,(m+\right.$ $1) T),(m+1) T \leq \tau)$. Since the system considered is a series one, the system will fail once one of the components fails. It leads from assumptions (3) and (5) that the defect of the failed component must have started at some time $u(u \in(m T,(m+1) T))$, the delay time is less than the interval $(m+1) T-u$, and another component is in normal or defective stage at the same time, as shown in Figure 1.

Therefore, the probability of such a failure renewal is given as

$$
\begin{aligned}
P\left(m T<T_{f}<(m+1) T\right)= & P\left(m T<X_{1}<(m+1) T, 0<Y_{1}<(m+1) T-X_{1}, X_{2}>X_{1}+Y_{1}\right) \\
& +P\left(m T<X_{1}<(m+1) T, 0<Y_{1}<(m+1) T-X_{1}, m T<X_{2}<X_{1}+Y_{1}, Y_{2}>X_{1}+Y_{1}-X_{2}\right) \\
& +P\left(m T<X_{2}<(m+1) T, 0<Y_{2}<(m+1) T-X_{2}, X_{1}>X_{2}+Y_{2}\right) \\
& +P\left(m T<X_{2}<(m+1) T, 0<Y_{2}<(m+1) T-X_{2}, m T<X_{1}<X_{2}+Y_{2}, Y_{1}>X_{2}+Y_{2}-X_{1}\right) \\
= & \int_{m T}^{(m+1) T} f_{1}(u) \int_{0}^{(m+1) T-u} g_{1}(v)\left[\left(1-F_{2}(u+v)\right)+\int_{m T}^{u+v} f_{2}(x) \int_{u+v-x}^{\infty} g_{2}(y) \mathrm{d} y \mathrm{~d} x\right] \mathrm{d} v \mathrm{~d} u \\
& +\int_{m T}^{(m+1) T} f_{2}(u) \int_{0}^{(m+1) T-u} g_{2}(v)\left[\left(1-F_{1}(u+v)\right)+\int_{m T}^{u+v} f_{1}(x) \int_{u+v-x}^{\infty} g_{1}(y) \mathrm{d} y \mathrm{~d} x\right] \mathrm{d} v \mathrm{~d} u, \quad 0 \leq m \leq m^{\prime} .
\end{aligned}
$$

Note that the failure renewal occurs prior to the swap, and the swap time $\tau=m^{\prime} T$, so $0 \leq m \leq m^{\prime}$. The first term in equation (3) corresponds to the probability that Component 1 fails and the second one is that of Component 2.
Similarly, the probability of failure renewal within $\left(T_{m}, T_{m}+z\right)$ :

$$
\begin{aligned}
P\left(m T<T_{f}<m T+z\right)= & \int_{m T}^{m T+z} f_{1}(u) \int_{0}^{m T+z-u} g_{1}(v)\left[+\int_{m T}^{u+v} f_{2}(x)\left(1-G_{2}(u+v-x)\right) \mathrm{d} x\right] \mathrm{d} v \mathrm{~d} u \\
& +\int_{m T}^{m T+z} f_{2}(u) \int_{0}^{m T+z-u} g_{2}(v)\left[\left(1-F_{1}(u+v)\right)\right. \\
& \left.+\int_{m T}^{u+v} f_{1}(x)\left(1-G_{1}(u+v-x)\right) \mathrm{d} x\right] \mathrm{d} v \mathrm{~d} u, \quad 0 \leq m \leq m^{\prime} .
\end{aligned}
$$

The failure renewal time, $Z$, is the time from a new system's operating to its first failure before the maximum usage time of the system. Its probability density function is a segment one on intervals $((m-1) T, m T)(1 \leq m \leq M+1)$. Let $f_{m}(z)$ be the probability density function of the time that $Z$ exceeds $(m-1) T$ in the scenario that the system fails at $T_{f}, T_{f} \in((m-1) T, m T)(1 \leq m \leq M+1)$, before which no defective stage has been identified by inspections. Then, by differentiating equation (4) with respect to $z$, we have

$$
\begin{aligned}
f_{m}(z)= & \int_{m T}^{m T+z} f_{1}(u)\left[\left(1-F_{2}(m T+z)\right)+\int_{m T}^{m T+z} f_{2}(x)\left(1-G_{2}(m T+z-x)\right) \mathrm{d} x\right] g_{1}(m T+z-u) \mathrm{d} u \\
& +\int_{m T}^{m T+z} f_{2}(x)\left[+\int_{m T}^{m T+z} f_{1}(u)\left(1-F_{1}(m T+z)\right)\right.
\end{aligned}
$$


The same derivation for the pdf will be used subsequently and it is omitted in the following for simplicity.

(2) The component is renewed at $T_{m+1}\left(T_{m+1} \leq \tau\right)$ due to the defect of component 1 or 2 . According to assumptions (3) and (4), in this scenario, one component's defect arrives at time $u\left(u \in\left(T_{m}, T_{m+1}\right)\right)$, its defective stage must be longer than $T_{m+1}-u$ and another component's duration time in the normal stage is longer than $T_{m+1}$, as shown in Figure 2. So, the probability is

$$
\begin{aligned}
P\left(T_{1 d}=(m+1) T\right)= & P\left(m T<X_{1}<(m+1) T, Y_{1}>(m+1) T-X_{1}, X_{2}>(m+1) T\right) \\
& +P\left(m T<X_{2}<(m+1) T, Y_{2}>(m+1) T-X_{2}, X_{1}>(m+1) T\right) \\
= & \left(1-F_{2}((m+1) T)\right) \int_{m T}^{(m+1) T} f_{1}(u)\left(1-G_{1}((m+1) T-u)\right) \mathrm{d} u \\
& +\left(1-F_{1}((m+1) T)\right) \int_{m T}^{(m+1) T} f_{2}(x)\left(1-G_{2}((m+1) T-x)\right) \mathrm{d} x, \quad 0 \leq m^{\prime} m^{\prime}-1 .
\end{aligned}
$$

(3) The component is renewed at $(m+1) T((m+1) T<\tau)$ due to the defects of components 1 and 2 . In this scenarios, the two components' defects occur between $m T$ and $(m+1) T$, and their defective stages must skip over $(m+1) T$; see Figure 3.

Then, we have the probability of such an event, according to the independency of the two components,

$$
\begin{aligned}
P\left(T_{2 d}=(m+1) T\right)= & P\left(\begin{array}{l}
m T<X_{1}<(m+1) T, Y_{1}>(m+1) T-X_{1}, \\
m T<X_{2}<(m+1) T, Y_{2}>(m+1) T-X_{2}
\end{array}\right) \\
= & \int_{m T}^{(m+1) T} f_{1}(u)\left(1-G_{1}((m+1) T-u)\right) \mathrm{d} u \\
& \int_{m T}^{(m+1) T} f_{2}(x)\left(1-G_{2}((m+1) T-x)\right) \mathrm{d} x, \quad 0 \leq m \leq m^{\prime}-1 .
\end{aligned}
$$

3.2. Probability of a Renewal after the Swap. Using a similar derivation to that in Section 3.1, we can obtain the following renewal probabilities after the swap. From assumption (4) and notations in Section 2, after the swap, the pdf and cdf of the duration time in defective stage for component $i(i=1,2)$ are $\widetilde{g}_{i}(u)$ and $\widetilde{G}_{i}(u)$, respectively. Furthermore, the lifetime, $T_{\text {age }}$, can be represented as $(M+1) T$.
(1) The fystem fails at $T_{f}$ $\left(T_{f} \in(m T,(m+1) T), m T \geq \tau\right)$. In this scenario, the duration times in the normal stage of the two components are longer than $\tau$, as shown in Figure 4 . So, the pdf of failure at $T_{f}$, $T_{f} \in(m T,(m+1) T), z \in(0, T), m T \geq \tau$ is given as

$$
\begin{aligned}
\tilde{f}_{m}(z)= & \lim _{\Delta z \longrightarrow 0} \frac{P\left(T_{f} \in(m T+z, m T+z+d z)\right)}{\Delta z} \\
= & \int_{m T}^{m T+z} f_{1}(u)\left[\left(1-F_{2}(m T+z)\right)+\int_{m T}^{m T+z} f_{2}(x)\left(1-\widetilde{G}_{2}(m T+z-x)\right) \mathrm{d} x\right] \\
& +\int_{m T}^{m T+z} f_{2}(x)\left[\left(1-F_{1}(m T+z)\right)+\int_{m T}^{m T+z} f_{1}(u)\left(1-\widetilde{G}_{1}(m T+z-u)\right) \mathrm{d} u\right] \widetilde{g}_{2}, \quad m^{\prime} \leq m \leq M .
\end{aligned}
$$


Similarly, the probability the system fails at $T_{s f}$ $\left(T_{s f} \in(m T,(m+1) T), m T \geq \tau\right)$ is

$$
\begin{aligned}
& P\left(m T<T_{s f}<(m+1) T\right) \\
& =\int_{m T}^{(m+1) T} f_{1}(u) \int_{0}^{(m+1) T-u} \widetilde{g}_{1}(v)\left[\left(1-F_{2}(u+v)\right)+\int_{m T}^{u+v} f_{2}(x) \int_{u+v-x}^{\infty} \widetilde{g}_{2}(y) \mathrm{d} y \mathrm{~d} x\right] \mathrm{d} v \mathrm{~d} u \\
& \quad+\int_{m T}^{(m+1) T} f_{2}(x) \int_{0}^{(m+1) T-x} \tilde{g}_{2}(y)\left[\left(1-F_{1}(x+y)\right)+\int_{m T}^{x+y} f_{1}(u) \int_{x+y-u}^{\infty} \tilde{g}_{1}(v) \mathrm{d} v \mathrm{~d} u\right] \mathrm{d} y \mathrm{~d} x, \quad m^{\prime} \leq m \leq M
\end{aligned}
$$

(2) The component is renewed at $(m+1) T$ $((m+1) T>\tau)$ due to the defect of component 1 or 2 ; see Figure 5.

$$
\begin{aligned}
P\left(T_{s 1 d}=(m+1) T\right)= & \left(1-F_{2}((m+1) T)\right) \int_{m T}^{(m+1) T} f_{1}(u)\left(1-\widetilde{G}_{1}((m+1) T-u)\right) \mathrm{d} u \\
& +\left(1-F_{1}((m+1) T)\right) \int_{m T}^{(m+1) T} f_{2}(x)\left(1-\widetilde{G}_{2}((m+1) T-x)\right) \mathrm{d} x, \quad m^{\prime} \leq m \leq M-1 .
\end{aligned}
$$

(3) The system is renewed at $T_{m+1}\left(T_{m+1}>\tau\right)$ due to the

The renewal probability in this scenario is defects of component 1 and 2; see Figure 6 .

$$
\begin{aligned}
P\left(T_{s 2 d}=(m+1) T\right) & =P\left(\begin{array}{l}
T_{m}<X_{1}<(m+1) T, Y_{1}>(m+1) T-X_{1}, \\
T_{m}<X_{2}<(m+1) T, Y_{2}>(m+1) T-X_{2}
\end{array}\right) \\
& =\int_{m T}^{(m+1) T} f_{1}(u)\left(1-\widetilde{G}_{1}((m+1) T-u)\right) \mathrm{d} u \\
& \int_{m T}^{(m+1) T} f_{2}(x)\left(1-\widetilde{G}_{2}((m+1) T-x)\right) \mathrm{d} x, \quad m^{\prime} \leq m \leq M-1 .
\end{aligned}
$$

(4) An overhaul is performed at $T_{\text {age }}$. According to the assumptions and notations in Section 2, when both components being normal at $T_{M}$ but not failed at
$T_{\text {age }}$, an overhaul will be performed at $T_{\text {age }}$; see Figure 7.

So, the overhaul probability is

$$
\begin{aligned}
P\left(\text { renewal at } T_{\text {age }}\right)= & P\left(X_{1}>T_{\text {age }}, X_{2}>T_{\text {age }}\right)+P\left(M T<X_{1}<T_{\text {age }}, \widetilde{Y}_{1}>T_{\text {age }}-X_{1}, X_{2}>T_{\text {age }}\right) \\
& +P\left(M T<X_{2}<T_{\text {age }}, \widetilde{Y}_{2}>T_{\text {age }}-X_{2}, X_{1}>T_{\text {age }}\right) \\
& +P\left(M T<X_{1}<T_{\text {age }}, \widetilde{Y}_{1}>T_{\text {age }}-X_{1}, M T<X_{2}<T_{\text {age }}, \widetilde{Y}_{2}>T_{\text {age }}-X_{2}\right) \\
= & \left(1-F_{1}\left(T_{\text {age }}\right)\right)\left(1-F_{2}\left(T_{\text {age }}\right)\right)+\left(1-F_{2}\left(T_{\text {age }}\right)\right) \int_{M T}^{T_{\text {age }}} f_{1}(u)\left(1-\widetilde{G}_{1}\left(T_{\text {age }}-u\right)\right) \mathrm{d} u \\
& +\left(1-F_{1}\left(T_{\text {age }}\right)\right) \int_{M T}^{T_{\text {age }}} f_{2}(x)\left(1-\widetilde{G}_{2}\left(T_{\text {age }}-x\right)\right) \mathrm{d} x \\
& +\int_{M T}^{T_{\text {age }}} f_{1}(u)\left(1-\widetilde{G}_{1}\left(T_{\text {age }}-u\right)\right) \mathrm{d} u \int_{M T}^{T_{\text {age }}} f_{2}(x)\left(1-\widetilde{G}_{2}\left(T_{\text {age }}-x\right)\right) \mathrm{d} x .
\end{aligned}
$$



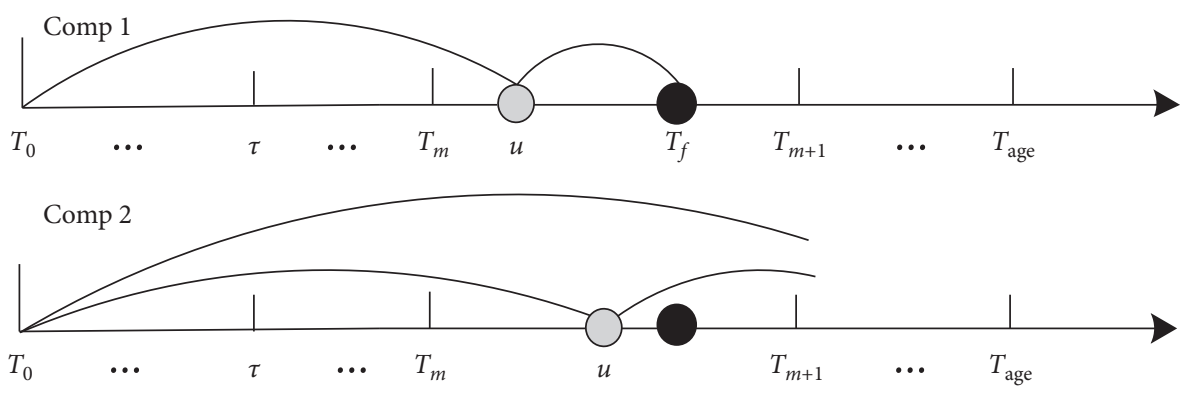

Figure 4: Illustration of the system failure after the swap.
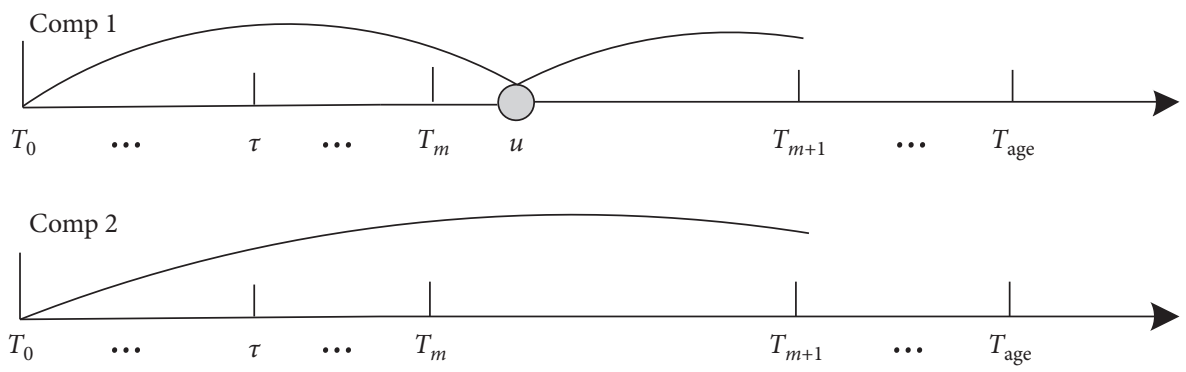

(a)
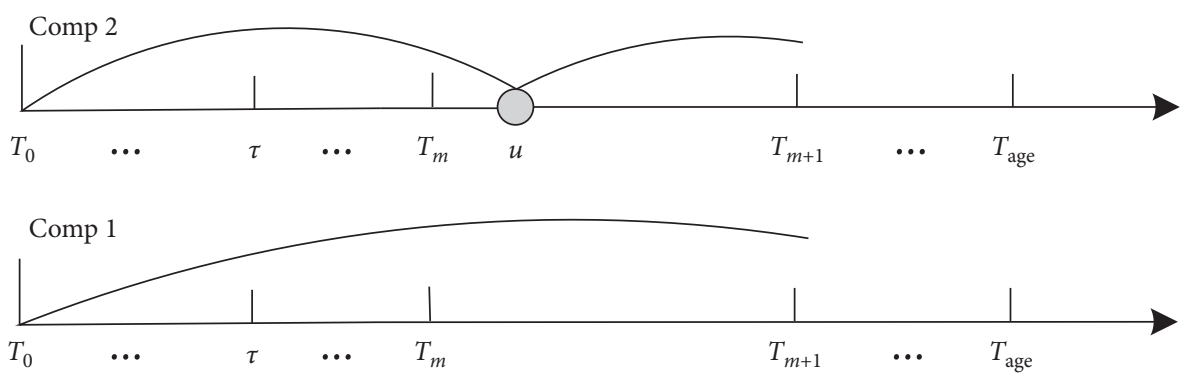

(b)

Figure 5: Illustration of a renewal after swap due to the defect of one component. (a) Component 1 is defective; component 2 is normal. (b) Component 2 is defective; component 1 is normal.

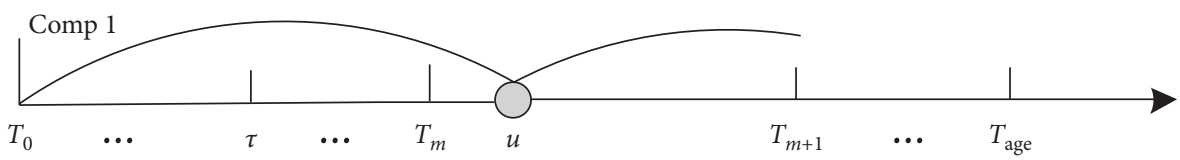

Comp 2

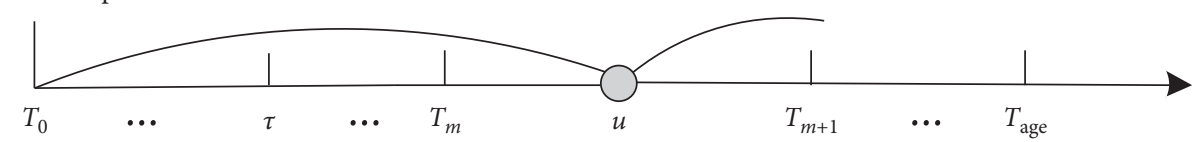

Figure 6: Illustration of a renewal after swap due to the defects of two components. 


\section{Cost Models}

4.1. The Expected Renewal Cycle Cost. Based on overhaul and different renewal probabilities formulated earlier and the costs due to inspections and replacements, we can obtain the expected renewal cycle cost.

The expected cost caused by a failure renewal $E\left(C_{\text {fail, M, } \tau}\right)$ is given by

$$
E\left(C_{\mathrm{fail}, M, \tau}\right)=\sum_{m=0}^{m^{\prime}-1}\left(m C_{i}+C_{f}\right) P\left(m T<T_{f}<(m+1) T\right)+\sum_{m=m^{\prime}}^{M}\left(m C_{i}+C_{f}\right) P\left(m T<T_{s f}<(m+1) T\right) .
$$

Using equations (4), (5), (9), and (10), the expected cost of an inspection renewal $E\left(C_{\mathrm{insp}, M, \tau}\right)$ is given by

$$
\begin{aligned}
E\left(C_{\text {insp }, M, \tau}\right)= & \sum_{m=0}^{m^{\prime}-1}\left[\begin{array}{c}
(m+1) C_{i}\left(P\left(T_{1 d}=(m+1) T\right)+P\left(T_{2 d}=(m+1) T\right)\right) \\
+C_{1 d} P\left(T_{1 d}=(m+1) T\right)+C_{2 d} P\left(T_{2 d}=(m+1) T\right)
\end{array}\right] \\
& +\sum_{m=m^{\prime}}^{M-1}\left[\begin{array}{c}
(m+1) C_{i}\left(P\left(T_{s 1 d}=(m+1) T\right)+P\left(T_{s 2 d}=(m+1) T\right)\right) \\
+C_{1 d} P\left(T_{s 1 d}=(m+1) T\right)+C_{2 d} P\left(T_{s 2 d}=(m+1) T\right)
\end{array}\right] .
\end{aligned}
$$

The expected cost of an overhaul is

$$
E\left(C_{\text {age }, M, \tau}\right)=\left(M C_{i}+C_{a}\right) P\left(\text { renewal at } T_{\text {age }}\right) .
$$

$$
\begin{aligned}
E\left(C_{\text {swap }, M, \tau}\right)= & C_{s} \sum_{m=m^{\prime}}^{M-1}\left(P\left(T_{s 1 d}=(m+1) T\right)+P\left(T_{s 2 d}=(m+1) T\right)\right) \\
& +C_{s} \sum_{m=m^{\prime}}^{M} P\left(m T<T_{s f}<(m+1) T\right)+C_{s} P\left(\text { renewal at } T_{\text {age }}\right) .
\end{aligned}
$$

4.2. The Expected Renewal Cycle Length. From the pdfs of a failure renewal, the expected length of a failure renewal $E\left(L_{\text {fail }}\right)$ is given by

$$
\begin{aligned}
E\left(L_{\mathrm{fail}, M, \tau}\right)= & \sum_{m=0}^{m^{\prime}-1} \int_{0}^{T}(m T+z) f_{m}(z) \mathrm{d} z \\
& +\sum_{m=m^{\prime}}^{M} \int_{0}^{T}(m T+z) \tilde{f}_{m}(z) \mathrm{d} z .
\end{aligned}
$$

$$
\begin{aligned}
E\left(L_{\mathrm{insp}, M, \tau}\right)= & \sum_{m=0}^{m^{\prime}-1}(m+1) T\left(P\left(T_{1 d}=(m+1) T\right)+P\left(T_{2 d}=(m+1) T\right)\right) \\
& +\sum_{m=m^{\prime}}^{M-1}(m+1) T\left(P\left(T_{s 1 d}=(m+1) T\right)+P\left(T_{s 2 d}=(m+1) T\right)\right) .
\end{aligned}
$$

According to the expected cycle cost and length with different renewals, the long run expected cost per unit time can be given using the renewal reward theorem (Ross [28], as shown in equation (18)): 

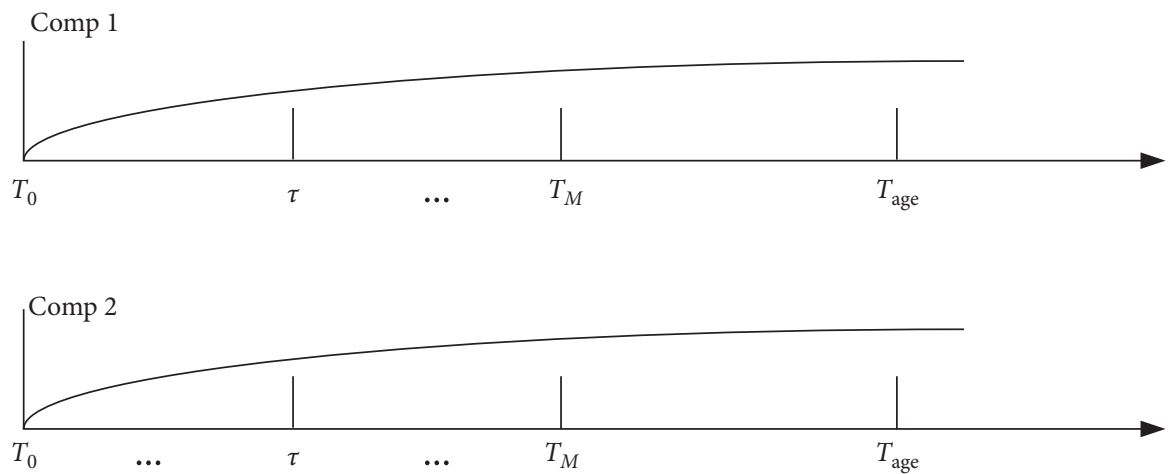

(a)
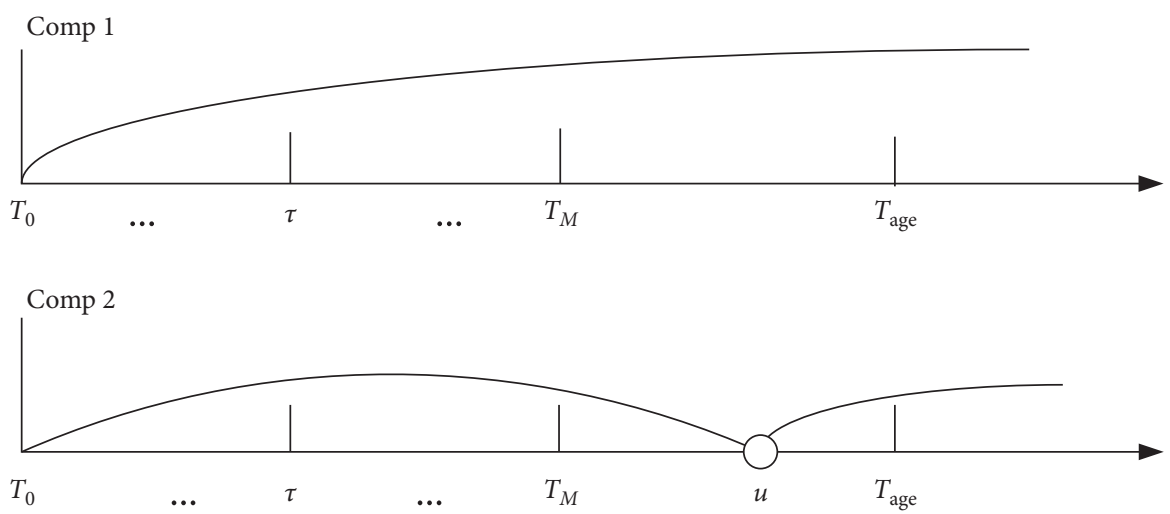

(b)
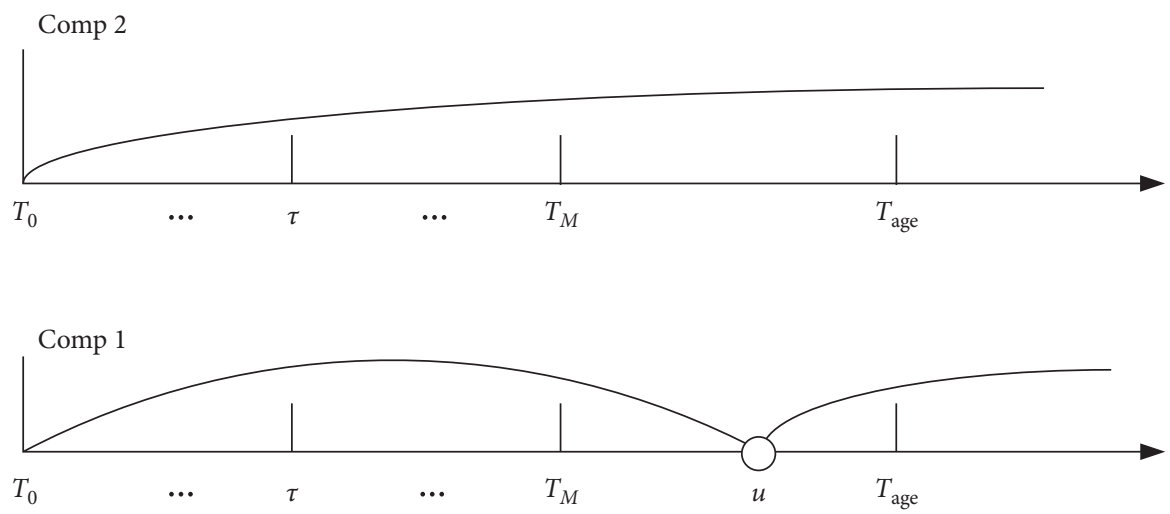

(c)
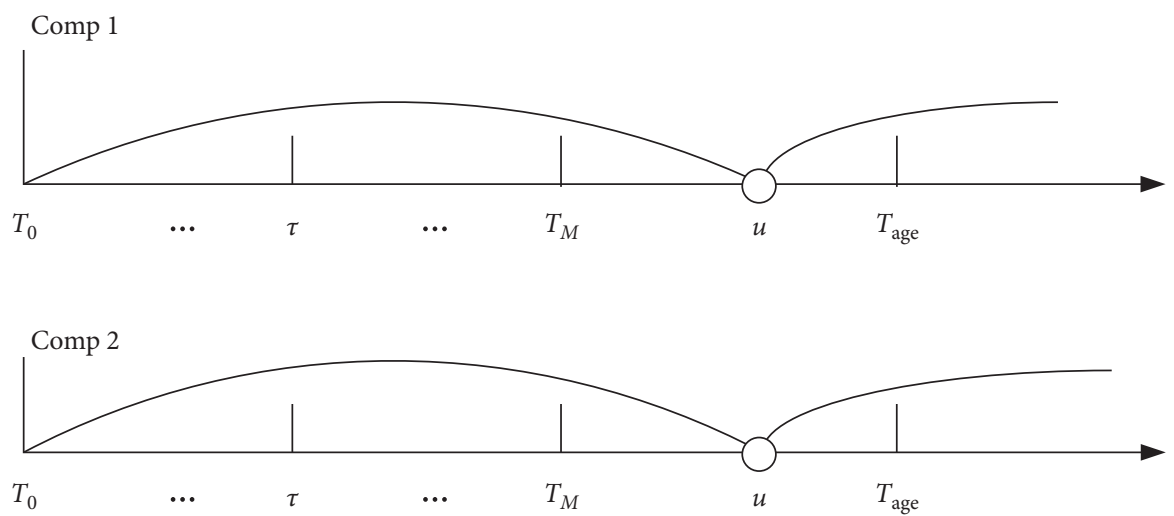

(d)

Figure 7: Illustration of an age renewal. 


$$
C(M, \tau)=\frac{E\left(C_{M, \tau}\right)}{E\left(L_{M, \tau}\right)}=\frac{E\left(C_{\text {fail }, M, \tau}\right)+E\left(C_{\text {insp }, M, \tau}\right)+E\left(C_{\text {age }, M, \tau}\right)+E\left(C_{\text {swap }, M, \tau}\right)}{E\left(L_{\text {fail }, M, \tau}\right)+E\left(L_{\text {insp }, M, \tau}\right)+T_{\text {age }} P\left(\text { renewal at } T_{\text {age }}\right)},
$$

where $E\left(C_{M, \tau}\right)$ is the expected cycle cost and $E\left(L_{M, \tau}\right)$ is the expected renewal cycle length.

We seek for the optimal inspection number $M$ and swap time $\tau$ which minimize equation (19).

\section{A Numerical Example}

5.1. Optimisation of the Number of Inspections and the Time of Swap. To solve equation (18), the hazard functions $\lambda_{i}(t)$ and $\widetilde{\lambda}_{i}(t)(i=1,2)$, the pdfs of $Y_{i}$ and $\widetilde{Y}_{i}(i=1,2), g_{i}(t)$ and $\widetilde{g}_{i}(t)$, must be available. From the literature in reliability, the time to failure of a component is popularly described as the Weibull distribution. So, we assume

$$
\begin{aligned}
& \lambda_{1}(t)=\frac{\beta^{(1)}}{\alpha^{(1)}}\left(\frac{t}{\alpha^{(1)}}\right)^{\beta^{(1)}-1}, \\
& \tilde{\lambda}_{1}(t)=\frac{\tilde{\beta}^{(1)}}{\widetilde{\alpha}^{(1)}}\left(\frac{t}{\widetilde{\alpha}^{(1)}}\right)^{\tilde{\beta}^{(1)}-1}, \\
& \lambda_{2}(t)=\frac{\mu^{(1)}}{v^{(1)}}\left(\frac{t}{\nu^{(1)}}\right)^{\mu^{(1)}-1}, \\
& \widetilde{\lambda}_{2}(t)=\frac{\widetilde{\mu}^{(1)}}{\widetilde{\nu}^{(1)}}\left(\frac{t}{\widetilde{\nu}^{(1)}}\right)^{\tilde{\mu}^{(1)}-1}, \\
& g_{1}(t)=\frac{\beta^{(2)}}{\alpha^{(2)}}\left(\frac{t}{\alpha^{(2)}}\right)^{\beta^{(2)}-1} e^{-\left(t / \alpha^{(2)}\right)^{\beta^{(2)}}}, \\
& \tilde{g}_{1}(t)=\frac{\tilde{\beta}^{(2)}}{\tilde{\alpha}^{(2)}}\left(\frac{t}{\widetilde{\alpha}^{(2)}}\right)^{\tilde{\beta}^{(2)}-1} e^{-\left(t \widetilde{\alpha}^{(2)}\right)^{\tilde{\beta}^{(2)}}}, \\
& g_{2}(t)=\frac{\mu^{(2)}}{v^{(2)}}\left(\frac{t}{\nu^{(2)}}\right)^{\mu^{(2)}-1} e^{-\left(t / v^{(2)}\right)^{\mu^{(2)}}}, \\
& \widetilde{g}_{2}(t)=\frac{\widetilde{\mu}^{(2)}}{\widetilde{v}^{(2)}}\left(\frac{t}{\widetilde{v}^{(2)}}\right)^{\widetilde{\mu}^{(2)}-1} e^{-\left(t \pi^{(2)}\right)^{\tilde{\mu}^{(2)}}},
\end{aligned}
$$

where $\alpha^{(1)}, \alpha^{(2)}, \widetilde{\alpha}^{(1)}, \widetilde{\alpha}^{(2)}, v^{(1)}, \widetilde{v}^{(1)}, v^{(2)}$, and $\widetilde{v}^{(2)}$ are scale parameters and $\beta^{(1)}, \beta^{(2)}, \widetilde{\beta}^{(1)}, \widetilde{\beta}^{(2)}, \mu^{(1)}, \widetilde{\mu}^{(1)}, \mu^{(2)}$, and $\widetilde{\mu}^{(2)}$ are shape parameters and they are bigger than 0 . The values of these parameters are listed in Tables 1 and 2 .
It can be shown from Table 1 that component 2 degrades more quickly than component 1 prior to the swap. While after the swap, the trend is the opposite. The other parameters of the system are listed in Table 3.

We first use the model presented in Section 3 based on the parameters in Tables 1 and 2. To analysis the effect of the component swap, we plot the figures of the maximal and minimal expected cost per unit time of equation (19) in terms of the inspection numbers in the cases that the swap is carried out and the expected cost per unit time in the case that the swap is not carried out, as shown in Figure 8. Note that for a given number of inspections $M$, the inspection interval $T=360 /(M+1)$, and the time of swap $\tau$ may be $m^{\prime} T\left(m^{\prime}=1,2, \ldots, M\right)$; the maximal and minimal expected cost per unit time are thus different. The figures indicate the joint optimization of number of inspections and swap time can decrease the expected cost per unit time. Detailed comparison analysis will be discussed in Section 5.2.

To obtain the optimal decision variables, the minimal expected cost per unit time in terms of the inspection numbers is shown in Figure 9. The figures indicate that the expected cost per unit time is minimized when the inspection number $M^{*}=11$ or $M^{*}=12$. So, the optimal inspection interval $T^{*}=360 /(11+1)=30$ or $T^{*}=360 /(12+1)=27.69$. Figure 10 is about the expected cost per unit time in terms of the swap time given $T^{*}=30$ or $T^{*}=27.69$. The figures indicate that when $m^{\prime}=5$, the expected cost per unit time is minimized. Hence, the optimal time of swap $\tau=5 T=150$ or $\tau=5 T=138.46$ with the expected cost per unit time of 0.01776 .

5.2. Comparison Analysis. For comparison, the long run expected cost per unit time without swap is given below.

According to model assumptions, the expected cost of a failure renewal in the case of no swap $E\left(C_{\text {fail }, M}\right)$ is given by

$$
E\left(C_{\text {fail }, M}\right)=\sum_{m=0}^{M}\left(M C_{i}+C_{f}\right) P\left(m T<T_{f}<(m+1) T\right),
$$

and the expected cost of an inspection renewal $E\left(C_{\text {insp }, M}\right)$ is given by

$$
\begin{aligned}
E\left(C_{\text {insp }, M}\right)= & \sum_{m=0}^{M}\left((m+1) C_{i}+C_{1 d}\right) P\left(T_{1 d}=(m+1) T\right) \\
& +\sum_{m=0}^{M}\left((m+1) C_{i}+C_{2 d}\right) P\left(T_{2 d}=(m+1) T\right) .
\end{aligned}
$$


TABLE 1: The parameters of hazard functions.

\begin{tabular}{|c|c|c|c|c|c|c|c|}
\hline \multicolumn{4}{|c|}{ Component 1} & \multicolumn{4}{|c|}{ Component 2} \\
\hline \multicolumn{2}{|c|}{$\lambda_{1}(t)$} & \multicolumn{2}{|c|}{$\widetilde{\lambda}_{1}(t)$} & \multicolumn{2}{|c|}{$\lambda_{2}(t)$} & \multicolumn{2}{|c|}{$\tilde{\lambda}_{2}(t)$} \\
\hline$\alpha^{(1)}$ & $\beta^{(1)}$ & $\widetilde{\alpha}^{(1)}$ & $\widetilde{\beta}^{(1)}$ & $v^{(1)}$ & $\mu^{(1)}$ & $\widetilde{v}^{(1)}$ & $\tilde{\mu}^{(1)}$ \\
\hline 260.83 & 1.27 & 260.83 & 1.32 & 220.61 & 1.45 & 220.61 & 1.3 \\
\hline
\end{tabular}

TABLE 2: The parameters of delay times.

\begin{tabular}{|c|c|c|c|c|c|c|c|}
\hline \multicolumn{4}{|c|}{ Component 1} & \multicolumn{4}{|c|}{ Component 2} \\
\hline$\alpha^{(2)}$ & $\beta^{(2)}$ & $\tilde{\alpha}^{(2)}$ & $\tilde{\beta}^{(2)}$ & $v^{(2)}$ & $\mu^{(2)}$ & $v^{(2)}$ & $\widetilde{\mu}^{(2)}$ \\
\hline 220.4 & 1.5 & 220.4 & 1.6 & 160.02 & 1.7 & 160.02 & 1.25 \\
\hline
\end{tabular}

TABLE 3: The other parameters of the system.

\begin{tabular}{ccccccc}
\hline$C_{i}$ & $C_{f}$ & $C_{1 d}$ & $C_{2 d}$ & $C_{s}$ & $C_{a}$ & \\
\hline 0.1 & 12 & 0.6 & 0.8 & 1 & 60 & \\
\hline
\end{tabular}

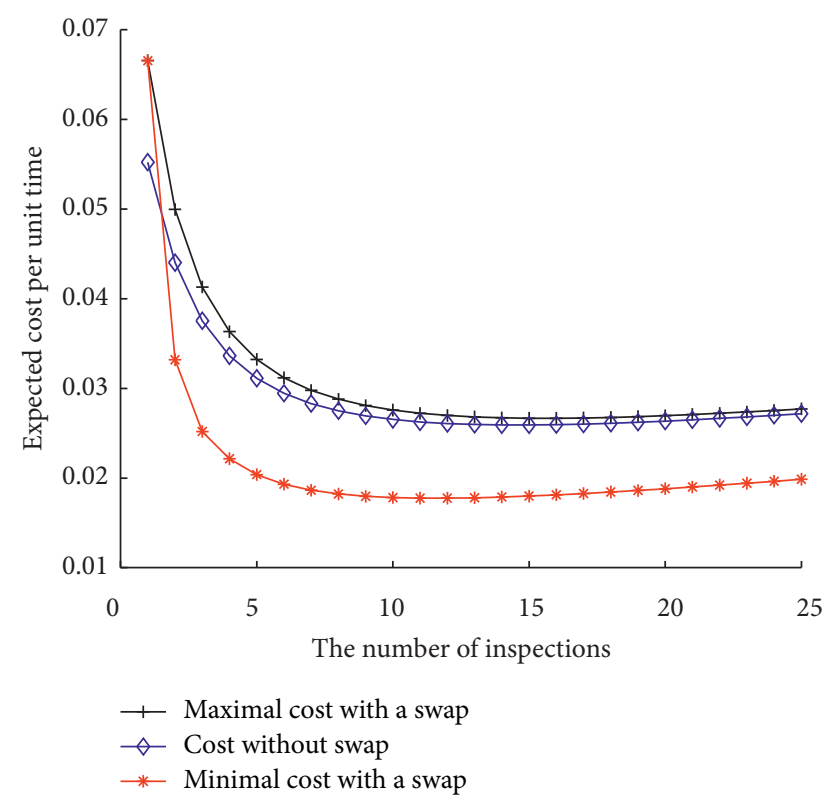

Figure 8: Illustration of the expected cost per unit time in terms of the number of inspections.

The expected cost of an overhaul in the case of no swap is

$$
E\left(C_{\text {age }, M}\right)=\left(M C_{i}+C_{a}\right) P\left(\text { renewal at } T_{\text {age }}\right) .
$$

The expected length of a failure renewal in the case of no swap $E\left(L_{\text {fail, } M}\right)$ is given by

$$
E\left(L_{\text {fail }, M}\right)=\sum_{m=0}^{M} \int_{0}^{T}(m T+z) f_{m}(z) \mathrm{d} z
$$

The expected length of an inspection renewal in the case of no swap is given as

$$
E\left(L_{\mathrm{insp}, M}\right)=\sum_{m=0}^{M}(m+1) T\left(P\left(T_{1 d}=(m+1) T\right)+P\left(T_{2 d}=(m+1) T\right)\right)
$$

Consequently, the long run expected cost per unit time can be given as 


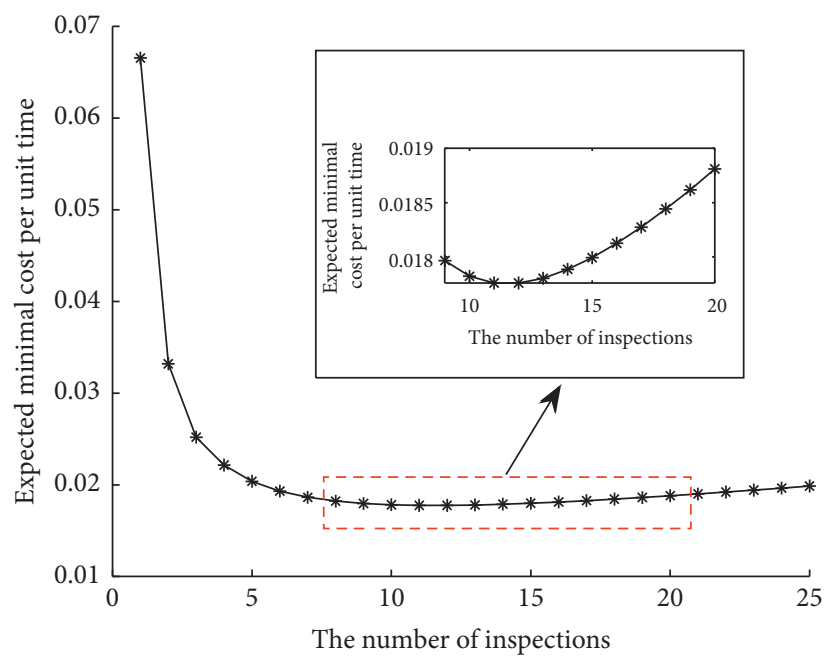

FIGURE 9: Illustration of the minimal expected cost per unit time in terms of the number of inspections.

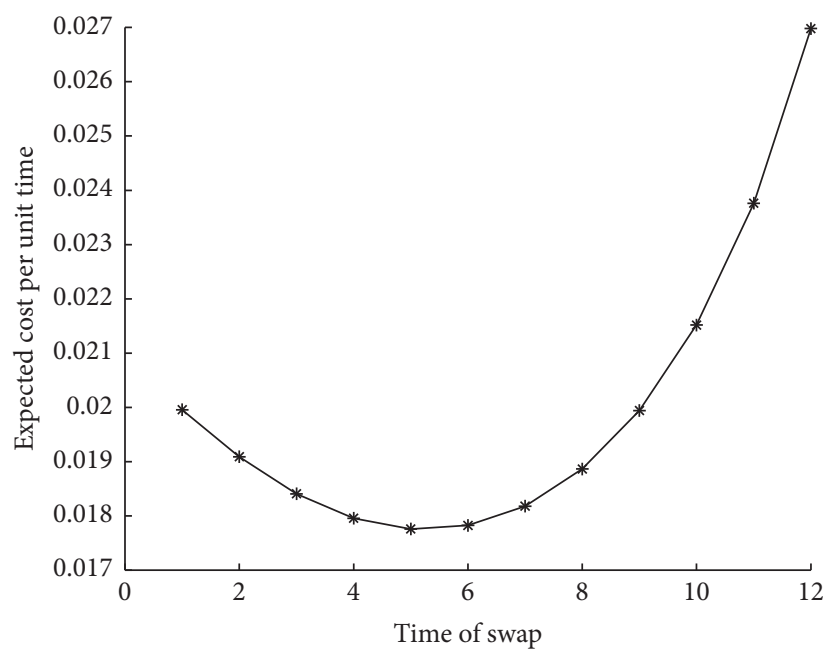

FiguRE 10: Illustration of the expected cost per unit time in terms of the time of swap given $M=12$.

$C_{M}=\frac{E\left(C_{M}\right)}{E\left(L_{M}\right)}=\frac{E\left(C_{\text {fail }, M}\right)+E\left(C_{\text {insp }, M}\right)+E\left(C_{\text {age }, M}\right)}{E\left(L_{\text {fail }, M}\right)+E\left(L_{\text {insp }, M}\right)+T_{\text {age }} P\left(\text { renewal at } T_{\text {age }}\right)}$.
Substituting parameters into the aforementioned formulas, the long run expected cost per unit time for the model without swap can be given (see Table 4). The table indicates that the optimal inspection number is 15 and the 
TABLE 4: Cost rates for various inspection numbers (without swap).

\begin{tabular}{lccc}
\hline$M$ & $E L_{M}$ & $E C_{M}$ & $C_{M}$ \\
\hline 1 & 252.1662 & 13.9284 & 0.0552 \\
2 & 211.5800 & 9.3132 & 0.0440 \\
3 & 190.1708 & 7.1397 & 0.0375 \\
4 & 177.3339 & 5.9638 & 0.0336 \\
5 & 168.8603 & 5.2563 & 0.0311 \\
6 & 162.8728 & 4.7979 & 0.0295 \\
7 & 158.4259 & 4.4848 & 0.0283 \\
8 & 154.9963 & 4.2629 & 0.0275 \\
9 & 152.2725 & 4.1016 & 0.0269 \\
10 & 150.0581 & 3.9822 & 0.0265 \\
11 & 148.2228 & 3.8930 & 0.0263 \\
12 & 146.6772 & 3.8261 & 0.0261 \\
13 & 145.3578 & 3.7763 & 0.0260 \\
$\mathbf{1 4}$ & $\mathbf{1 4 4 . 2 1 8 6}$ & $\mathbf{3 . 7 3 9 6}$ & $\mathbf{0 . 0 2 5 9}$ \\
$\mathbf{1 5}$ & $\mathbf{1 4 3 . 2 2 5 2}$ & 3.7134 & $\mathbf{0 . 0 2 5 9}$ \\
16 & 142.3511 & 3.6955 & 0.0260 \\
17 & 141.5763 & 3.6844 & 0.0260 \\
18 & 140.8847 & 3.6790 & 0.0261 \\
19 & 140.2636 & 3.6782 & 0.0262 \\
20 & 139.7027 & 3.6814 & 0.0264 \\
21 & 139.1937 & 3.6879 & 0.0265 \\
22 & 138.7298 & 3.6973 & 0.0267 \\
23 & 138.3052 & 3.7092 & 0.0268 \\
24 & 137.9152 & 3.7232 & 0.0270 \\
25 & 137.5556 & 3.7391 & 0.0272 \\
\hline
\end{tabular}

TABle 5: Optimum policy for various $C_{s}$.

\begin{tabular}{lccc}
\hline$C_{s}$ & $M^{*}$ & $\tau^{*}$ & $C\left(M^{*}, \tau^{*}\right)$ \\
\hline 2 & 15 & 157.5 & 0.02082 \\
3 & 15 & 157.5 & 0.02326 \\
4 & 16 & 169.4 & 0.02527 \\
5 & 16 & 169.4 & 0.02672 \\
6 & 17 & 200.0 & 0.02779 \\
\hline
\end{tabular}

corresponding cost rate is 0.02593 , which is greater than 0.01776 , the cost rate in the case of swap.

As the long run expected cost rate of the swap model increases with the increasing of the extra cost for the swap, the maintenance police proposed in this paper may have lost the economic efficiency when the extra cost for the swap is large enough. We will discuss this problem by a numerical method.

Let $C_{s}$ change from 2 to 6 by step 1 and the other system parameters be the same as those in Section 5.1. The optimal strategies $\left(M^{*}, \tau^{*}\right)$ and their corresponding expected cost rates $C\left(M^{*}, \tau^{*}\right)$ are presented in Table 5 .

The table indicates the cost rate for $C_{s}=4$ is smaller than 0.025927 , the cost rate for the model without swap. While for $C_{s}=5$, the cost rate is greater than 0.025927. So, the threshold for the cost of the swap so that no benefit is derived is between 4 and 5 .

\section{Conclusions and Further Research}

In this paper, we develop a 2-component swappable series system model based on a two-stage failure process characterized by the delay-time concept. The system is subjected to periodic inspections at an interval and the two components can be swapped once at some epoch that an inspection is carried out. Our objective is to optimize the inspection interval and the time of swap jointly by minimizing the expected cost per unit time. The motivation of the system comes from the performance process of steelmaking converter system and the maintenance of vehicles and railway lines. The results obtained in this paper may be useful in improving the economic efficiency of these systems. The numerical example demonstrates the results of the proposed model. Economic efficiency of the proposed model is stated by comparison analysis.

There are several further research topics which will be studied in the future. First, the number of swaps should be optimized jointly with the inspection interval and the time of swap. Second, imperfect inspection, repairs, and PMs should be discussed. Third, the parameters estimations of the Weibull distributions and how to describe relationships of two failure processes, prior to and after swap, are also important topics.

\section{Data Availability}

The data used to support the findings of this study are included within the article.

\section{Conflicts of Interest}

The authors declare that they have no conflicts of interest regarding the publication of this paper.

\section{Acknowledgments}

This work was supported partly by the Universities in Hebei Province Science and Technology Research Project (ZD2018073).

\section{References}

[1] A. Almuhtady, S. Lee, E. Romeijn, M. Wynblatt, and J. Ni, “A degradation-informed battery-swapping policy for fleets of electric or hybrid-electric vehicles," Transportation Science, vol. 48, no. 4, pp. 609-618, 2014.

[2] Y. Fu, T. Yuan, and X. Zhu, "Optimum periodic component reallocation and system replacement maintenance," IEEE Transactions on Reliability, vol. 68, no. 2, pp. 753-763, 2019.

[3] Q. Sun, Z. S. Ye, and X. Zhu, "Managing component degradation in series systems for balancing degradation through reallocation and maintenance," IIE Transactions, vol. 52, pp. 1-34, 2019.

[4] A. H. Christer, "Innovative decision making," in Proceedings of the NATO Conference on the Role and Effectiveness of Theories of Decision in Practice, K. C Bowen and D. J. White, Eds., pp. 368-377, Hodder and Stoughton, London, UK, 1976.

[5] A. H. Christer and W. M. Waller, "Reducing production downtime using delay-time analysis," Journal of the Operational Research Society, vol. 35, no. 6, pp. 499-512, 1984.

[6] W. Wang, "An inspection model based on a three-stage failure process," Reliability Engineering \& System Safety, vol. 96, no. 7, pp. 838-848, 2011. 
[7] W. Wang, "An overview of the recent advances in delay-timebased maintenance modelling," Reliability Engineering \& System Safety, vol. 106, no. 5, pp. 165-178, 2012.

[8] W. Wang, F. Zhao, and R. Peng, "A preventive maintenance model with a two-level inspection policy based on a threestage failure process," Reliability Engineering \& System Safety, vol. 121, no. 1, pp. 207-220, 2014.

[9] L. Yang, X. Ma, Q. Zhai, and Y. Zhao, "A delay time model for a mission-based system subject to periodic and random inspection and postponed replacement," Reliability Engineering \& System Safety, vol. 150, pp. 96-104, 2016.

[10] R. D. Baker and W. Wang, "Developing and testing the delaytime model," Journal of the Operational Research Society, vol. 44, no. 4, pp. 361-374, 1993.

[11] R. D. Baker and A. H. Christer, "Review of delay-time OR modelling of engineering aspects of maintenance," European Journal of Operational Research, vol. 73, no. 3, pp. 407-422, 1994.

[12] X. Jia and A. H. Christer, "A periodic testing model for a preparedness system with a defective state," IMA Journal of Management Mathematics, vol. 13, no. 1, pp. 39-49, 2002.

[13] W. Wang, "A delay time based approach for risk analysis of maintenance activities," The Journal of the Safety and Reliability Society, vol. 23, no. 1, pp. 103-113, 2003.

[14] T. Aven and I. T. Castro, "A delay-time model with safety constraint," Reliability Engineering \& System Safety, vol. 94, no. 2, pp. 261-267, 2009.

[15] B. Jones, I. Jenkinson, and J. Wang, "Methodology of using delay-time analysis for a manufacturing industry," Reliability Engineering \& System Safety, vol. 94, no. 1, pp. 111-124, 2009.

[16] P. A. Scarf, C. Cavalcante, R. A. Dwight, and P. Gordon, "An age-based inspection and replacement policy for heterogeneous components," IEEE Transactions on Reliability, vol. 58, no. 4, pp. 641-648, 2009.

[17] W. Wang, D. Banjevic, and M. Pecht, "A multi-component and multi-failure mode inspection model based on the delay time concept," Reliability Engineering \& System Safety, vol. 95, no. 8, pp. 912-920, 2010.

[18] X. Lu, W. Wang, H. Yang, M. J. Zuo, and D. Zhou, "Optimizing the periodic inspection interval for a 1-out-of-2 cold standby system using the delay-time concept," Quality and Reliability Engineering International, vol. 28, no. 6, pp. 648662, 2012.

[19] X. Liu, W. Wang, and R. Peng, "An integrated production, inventory and preventive maintenance model for a multiproduct production system," Reliability Engineering \& System Safety, vol. 137, no. 2, pp. 76-86, 2015.

[20] X. Ma, W. Wang, X. Liu, and R. Peng, "Optimal inspection and replacement strategy for systems subject to two types of failures with adjustable inspection intervals," Journal of Shanghai Jiaotong University (Science), vol. 22, no. 6, pp. 752-755, 2017.

[21] R. Peng, B. Liu, Q. Zhai, and W. Wang, "Optimal maintenance strategy for systems with two failure modes," Reliability Engineering \& System Safety, vol. 188, pp. 624-632, 2019.

[22] L. Yang, X. Ma, R. Peng, Q. Zhai, and Y. Zhao, “A preventive maintenance policy based on dependent two-stage deterioration and external shocks," Reliability Engineering \& System Safety, vol. 160, pp. 201-211, 2017.

[23] Q. Qiu and L. Cui, "Optimal mission abort policy for systems subject to random shocks based on virtual age process," Reliability Engineering \& System Safety, vol. 189, pp. 11-20, 2019.
[24] X. Zhao, J. Sun, Q. Qiu, and K. Chen, "Optimal inspection and mission abort policies for systems subject to degradation," European Journal of Operational Research, vol. 292, no. 2, pp. 610-621, 2020.

[25] Q. Qiu and L. Cui, "Reliability evaluation based on a dependent two-stage failure process with competing failures," Applied Mathematical Modelling, vol. 64, pp. 699-712, 2018.

[26] M. Heydari, "Two-stage failure modeling and optimal periodic inspection policy during the extended warranty period," International Journal of Management Science and Engineering Management, vol. 14, no. 4, pp. 264-272, 2019.

[27] L. Wang, Y. Yang, H. Zhu, and G. Liu, "Optimal conditionbased renewable warranty policy for products with three-stage failure process," Quality Technology \& Quantitative Management, vol. 17, no. 2, pp. 216-233, 2020.

[28] S. M. Ross, Stochastic Process, John Wiley \& Sons, New York, NY, USA, 1983. 